\title{
The in vitro leishmanicidal activity of hexadecylphosphocholine (miltefosine) against four medically relevant Leishmania species of Brazil
}

\author{
Eliane de Morais-Teixeira', Quesia Souza Damasceno', \\ Mariana Kolos Galuppo', Alvaro José Romanha², Ana Rabello'/+ \\ ${ }^{1}$ Laboratório de Pesquisas Clínicas ${ }^{2}$ Laboratório de Parasitologia Celular e Molecular, \\ Centro de Pesquisas René Rachou-Fiocruz, Av. Augusto de Lima 1715, 30190-002 Belo Horizonte, MG, Brasil
}

The in vitro leishmanicidal activity of miltefosine ${ }^{\circledR}$ (Zentaris GmbH) was assessed against four medically relevant Leishmania species of Brazil: Leishmania (Leishmania) amazonensis, Leishmania (Viannia) braziliensis, Leishmania (Viannia) guyanensis and Leishmania (Leishmania) chagasi. The activity of miltefosine against these New World species was compared to its activity against the Old World strain, Leishmania (Leishmania) donovani, which is known to be sensitive to the effects of miltefosine. The $I C_{50}$ and $I C_{90}$ results suggested the New World species harboured similar in vitro susceptibilities to miltefosine; however, miltefosine was approximately 20 times more active against the Old World L. (L.) donovani than against the New World L. (L.) chagasi species. The selectivity index varied from 17.2-28.9 for the New World Leishmania species and up to 420.0 for L. (L.) donovani. The differences in susceptibility to miltefosine suggest that future clinical trials with this drug should include a laboratory pre-evaluation and a dose-defining step.

Key words: Leishmania - miltefosine - in vitro drug evaluation

Reducing the lethality and morbidity of leishmaniasis relies primarily on chemotherapeutic treatment. Currently, the clinically available drugs have significant shortcomings, including high toxicity, long therapeutic regimens, parenteral administration and high cost.

Miltefosine ${ }^{\circledR}$ (Zentaris $\mathrm{GmbH}$ ), an alkylphosphocoline, was introduced in the last decade as an alternative oral drug for the treatment of visceral leishmaniasis. It was initially developed as an anticancer agent that interferes with the apoptotic and sterol biosynthesis pathways (Oullette et al. 2004, Paris et al. 2004). Both in vitro and in vivo studies have shown that miltefosine is active against Leishmania (Leishmania) donovani (Croft et al. 1996, Le Fichoux et al. 1998). Clinical trials in India have demonstrated a cure rate of $97 \%$ among patients treated for visceral leishmaniasis with oral miltefosine at a dose of $2.5 \mathrm{mg} / \mathrm{Kg} /$ day for four weeks (Sundar et al. 1998, Bhattacharya et al. 2004). This drug has also been tested for the treatment of cutaneous leishmaniasis in South America. In Colombia, where Leishmania panamensis is prevalent, the efficacy of miltefosine was $91 \%$ compared to a cure rate of $38 \%$ in the placebo group. In Guatemala, where Leishmania (Viannia) braziliensis and Leishmania (Leishmania) mexicana are prevalent, a cure rate of $53 \%$ with miltefosine was observed (Soto et al. 2004). Miltefosine has also been successfully used for treating HIV-1 patients who presented with diffuse cutaneous leishmaniasis caused by Leishmania major (Schraner et al. 2005).

Financial support: FAPEMIG, FIOCRUZ, CNPq

+ Corresponding author: ana@cpqrr.fiocruz.br

Received 31 January 2011

Accepted 3 May 2011
In addition to its efficacy in treating leishmaniasis, miltefosine is also the only drug with leishmanicidal activity that can be effectively administered orally. However, the side effects of vomiting and diarrhoea as well as the drugs documented hazards, including teratogenic effects in animals (Kaminsky 2002) and increased blood levels of transaminase, urea and creatinine (Fischer et al. 2001), limit its overall use.

An important intrinsic variation in miltefosine susceptibility of Leishmania clinical isolates was observed for visceral leishmaniasis in Nepal and cutaneous leishmaniasis in Peru (Yardley et al. 2005).

Knowledge of this intrinsic variation in the drug susceptibility of prevalent species of Leishmania may help define drug schedules in clinical trials that aim to evaluate the efficacy of miltefosine in different species. The present study evaluates the in vitro susceptibility of four medically important Leishmania species found in Brazil to miltefosine and compares the susceptibility of these species to that of $L$. (L.) donovani.

\section{MATERIALS AND METHODS}

Drugs - Miltefosine was a gift from Zentaris $\mathrm{GmbH}$ (USA). For the in vitro assays, stock solutions were prepared by diluting miltefosine in deionised water and the solutions were stored at $-20^{\circ} \mathrm{C}$ until use. Amphotericin B deoxycholate $(\mathrm{AmB})\left(\right.$ Cristalia $^{\circledR}$ - Produtos Químicos Farmacêuticos Ltda, Brazil) was used as an internal control at a concentration of $0.2 \mu \mathrm{g} / \mathrm{mL}$. All subsequent dilutions for miltefosine and AmB were freshly made in Roswell Park Memorial Institute (RPMI) 1640 media (Sigma-Aldrich, St. Louis, MO, USA).

Leishmania strains and the cultivation of amastigote-like forms - A panel of strains, including the Leishmania (Leishmania) chagasi strain MHOM/BR/70/ BH46, Leishmania (Leishmania) amazonensis strain 
IFLA/BR/1967/PH-8, L. (V.) braziliensis strain WHOMHOM/BR/75/M2903, Leishmania (Viannia) guyanensis strain MHOM/BR/1997/321-P and L. (L.) donovani strain MHOM/ET/1967/HU3 was used throughout the study. The strains were maintained by successive passaging in golden hamsters (Mesocricetus auratus). Amastigotes were harvested from the spleens of animals infected with $L$. (L.) chagasi or $L$. (L.) donovani or from the skin lesions of animals infected with $L$. (L.) amazonensis, $L$. (V.) braziliensis or L. (V.) guyanensis.

Promastigotes of $L$. (L.) chagasi, L. (L.) amazonensis and $L$. (V.) guyanensis were cultivated at $26^{\circ} \mathrm{C}$ in Schneider's medium supplemented with $10 \%$ heatinactivated fetal bovine serum (FBS) (Gibco, Grand Island, NY, USA), penicillin (100 U/mL) and streptomycin $(100 \mu \mathrm{g} / \mathrm{mL})$. L. (L.) braziliensis and $L$. (L.) donovani were cultivated at $26^{\circ} \mathrm{C}$ in biphasic medium Novy, McNeal and Nicolle/liver infusion tryptose supplemented with antibiotics penicillin $(100 \mathrm{U} / \mathrm{mL})$ and streptomycin $(100 \mu \mathrm{g} / \mathrm{mL})$ and $10 \%$ FBS. Amastigotelike forms were transformed from stationary phase promastigotes. Promastigotes were centrifuged (1,000 $\mathrm{g}$ for $10 \mathrm{~min}$ at $4^{\circ} \mathrm{C}$ ), resuspended in Schneider's medium and transformed into amastigote-like forms according to a previously established protocol (Morais-Teixeira et al. 2008). The conditions for transforming $L$. (V.) guyanensis were the same as those for $L$. (V.) braziliensis and $L$. (L.) amazonensis. After six days of culture, stationary promastigote forms of $L$. (L.) donovani were used in the macrophage assays. Following additional experimentation, we found no differences between the promastigotes and the amastigote-like $L$. $(L$.) donovani in the murine macrophage infections.

Cytotoxicity test - The Alamar Blue $\mathrm{TM}^{\mathrm{TM}}$ micromethod was used to estimate the $50 \%$ cytotoxicity concentration $\left(\mathrm{CC}_{50}\right)$ of miltefosine in macrophages. Briefly, peritoneal macrophages from Balb/c mice were harvested and washed by centrifugation with ice-cold RPMI 1640 medium. The macrophages were resuspended at a density of $1 \times 10^{6} \mathrm{cells} / \mathrm{mL}$. Aliquots of $100 \mu \mathrm{L}$ were plated in 96-well tissue culture plates at a cellular density of $1 \mathrm{x}$ $10^{5}$ macrophages/well. The cells were allowed to adhere to the culture plates overnight at $37^{\circ} \mathrm{C}$ with $5 \% \mathrm{CO}_{2}$. The macrophages were incubated with a 10 point standard curve using two-fold serial dilutions of miltefosine with concentrations between $0.05 \mu \mathrm{g} / \mathrm{mL}$ and $1000 \mu \mathrm{g} / \mathrm{mL}$ $(0.012 \mu \mathrm{M}$ and $2453.51 \mu \mathrm{M})$. After $68 \mathrm{~h}$ of incubation, $10 \mu \mathrm{L}$ of Alamar Blue ${ }^{\mathrm{TM}}$ was added to each well and the plates were further incubated for an additional $4 \mathrm{~h}$.

The absorbance of 570 and $600 \mathrm{~nm}$ were measured simultaneously. The optical density at 570 and $600 \mathrm{~nm}$ was read with a 96-well scanner (Spectra Max M). Three independent experiments were performed in triplicate to determine the $\mathrm{CC}_{50}$ of miltefosine. The optical density in the absence of the drug was set as the $100 \%$ control value. The selectivity index (SI) was determined based on the equation $\mathrm{CC}_{50}$ /inhibitory concentration $\left(\mathrm{IC}_{50}\right)$ as described by Weninger et al. (2001).

Amastigote-macrophage assay - Balb/c mice were injected intraperitoneally with $1.5 \mathrm{~mL}$ of a $3 \%$ thioglycolate medium (Biobrás, Brazil). After $72 \mathrm{~h}$, the peri- toneal macrophages were harvested and washed with cold RPMI 1640 medium. The macrophages were then counted, centrifuged and resuspended at a concentration of $4 \times 10^{5}$ cells $/ \mathrm{mL}$ in RPMI-1640 medium without supplementation. Sterile round glass coverslips $(13 \mathrm{~mm})$ were placed in 24-well culture plates. In each well, $500 \mu \mathrm{L}$ of the macrophage solution was allowed to attach to the coverslips for $2 \mathrm{~h}$ at $37^{\circ} \mathrm{C}$ in $5 \% \mathrm{CO}_{2}$. The medium was then discarded and replaced with $500 \mu \mathrm{L}$ of fresh RPMI medium containing $10 \%$ FBS, penicillin $(50 \mathrm{U} / \mathrm{mL})$ and streptomycin $(50 \mu \mathrm{g} / \mathrm{mL})$ at $37^{\circ} \mathrm{C}$.

On the following day, a suspension of $4 \times 10^{6}$ amastigote-like $L$. (L.) chagasi, $L$. (L.) amazonensis, $L$. (V.) braziliensis or $L$. (V.) guyanensis and for $L$. (L.) donovani promastigote forms at stationary phase were added to each well in $500 \mu \mathrm{L}$ of RPMI with a final macrophage to parasite ratio of 1:10. The plates were incubated for $4 \mathrm{~h}$ at $37^{\circ} \mathrm{C}$ in $5 \% \mathrm{CO}_{2}$. The medium was then aspirated to remove free-floating parasites. Fresh RPMI $(1 \mathrm{~mL})$ was added with the following miltefosine concentrations: $8.0 \mu \mathrm{g} / \mathrm{mL}-0.19 \mu \mathrm{g} / \mathrm{mL}(19.6 \mu \mathrm{M}-0.46 \mu \mathrm{M})$ for L. (V.) braziliensis, $10.0 \mu \mathrm{g} / \mathrm{mL}-0.16 \mu \mathrm{g} / \mathrm{mL}(24.53 \mu \mathrm{M}-0.39 \mu \mathrm{M})$ for L. (V.) guyanensis and L. (L.) chagasi, $5.0 \mu \mathrm{g} / \mathrm{mL}-0.078$ $\mu \mathrm{g} / \mathrm{mL}(12.27 \mu \mathrm{M}-0.19 \mu \mathrm{M})$ for $L$. (L.) amazonensis and $1.0 \mu \mathrm{g} / \mathrm{mL}-0.016 \mu \mathrm{g} / \mathrm{mL}(2.45 \mu \mathrm{M}-0.039 \mu \mathrm{M})$ for $L$. (L.) donovani $)$. AmB $(0.2 \mu \mathrm{g} / \mathrm{mL})$ and fresh medium without drug were also added to separate triplicate wells. After $72 \mathrm{~h}$ incubation at $37^{\circ} \mathrm{C}$ in $5 \% \mathrm{CO}_{2}$, the medium was aspirated and the coverslips were removed, air-dried and glued to microscope slides. Following staining with Giemsa, the cells were counted. The assay results were analysed if at least $80 \%$ of the macrophages in the control wells were infected. Three independent experiments in triplicate were performed for each drug concentration to determine the miltefosine efficacy. The results were presented as the ratio of infection (number of amastigotes) between the treated and non-treated macrophage cultures. The results were expressed as inhibitory concentrations $\left(\mathrm{IC}_{50}\right.$ and $\left.\mathrm{IC}_{90}\right)$. The animals used in this study were handled according to local and federal regulations and the research protocols were approved by the Fiocruz Committee on Animal Research (Protocol P-321/06, license L-0024/8).

Data analysis - The statistical analysis was performed using MINITAB V. 13.1 or GraphPad Prism 4 (GraphPad Software, San Diego, CA, USA). The activities of miltefosine among the Leishmania species were compared using an analysis of variance and $p$ value $\leq$ 0.05 was considered to be significant. The $\mathrm{IC}_{50}, \mathrm{IC}_{90}$ and $\mathrm{CC}_{50}$ were calculated using linear regression analysis or interpolation (MiniTab 13.0 or Microsoft Office Excel 2003) (Huber \& Koella 1993). Linear regression was used when the distribution was normal (parametric method) and linear interpolation was applied when the distribution was not normal (non-parametric).

\section{RESULTS AND DISCUSSION}

The activity of miltefosine against the five Leishmania species is shown in Table. No statistically significant differences were observed between the $\mathrm{IC}_{50}$ values determined for the four evaluated New World Leishmania species $(\mathrm{p}=0.295)$. In contrast, Escobar et al. (2002), 
TABLE

Inhibitory concentrations ( $\mathrm{IC}_{50}$ and $\mathrm{IC}_{90}$ ) and selectivity index (SI) of miltefosine against intracellular amastigotes of five Leishmania species

\begin{tabular}{|c|c|c|c|c|c|c|c|}
\hline \multirow[b]{2}{*}{ Leishmania $\mathrm{spp}$} & \multicolumn{2}{|r|}{$\mathrm{IC}_{50}$} & \multicolumn{2}{|r|}{$\mathrm{IC}_{90}$} & \multirow{2}{*}{$\begin{array}{c}\text { Infection } \\
\operatorname{level}^{b}[\%( \pm \mathrm{SD})]\end{array}$} & \multirow[b]{2}{*}{$\mathrm{SI}^{c}$} & \multirow[b]{2}{*}{$\mathrm{R}^{2}$} \\
\hline & $\mu \mathrm{M}$ & $\mu \mathrm{g} / \mathrm{mL}\left(\mathrm{CI}^{a}\right)$ & $\mu \mathrm{M}$ & $\mu \mathrm{g} / \mathrm{mL}\left(\mathrm{CI}^{a}\right)$ & & & \\
\hline L. (L.) amazonensis & 3.21 & $1.31(0.75-1.89)$ & 7.88 & $3.21(2.19-4.24)$ & $84.3(4.5)$ & 28.85 & 0.798 \\
\hline L. (V.) braziliensis & 5.40 & $2.20(1.54-2.90)$ & 13.91 & $5.67(4.67-6.67)$ & $81(0.5)$ & 17.18 & 0.765 \\
\hline L. (V.) guyanensis & 4.02 & 1.64 & 14.84 & 6.05 & $84.2(2.6)$ & 26.05 & $-{ }^{d}$ \\
\hline L. (L.) chagasi & 4.46 & $1.82(0.65-3.00)$ & 12.39 & $5.05(3.27-6.82)$ & $83.1(3.0)$ & 20.77 & 0.713 \\
\hline L. (L.) donovani & 0.22 & $0.09(0.03-0.014)$ & 0.52 & $0.21(0.14-0.27)$ & $80(0.0)$ & 420.0 & 0.700 \\
\hline
\end{tabular}

$a$ : confidence interval (CI) 95\%; $b$ : infection level indicates the percentage of macrophages infected in untreated control culture; $c$ : selectivity index $(\mathrm{SI})=\mathrm{CC}_{50} / \mathrm{IC}_{50} ; d$ : $\mathrm{IC}_{50}$ and $\mathrm{IC}_{90}$ determined by linear interpolation; $\mathrm{R}^{2}$ : coefficient of linear regression; SD: standard deviation.

Yardley et al. (2005) and Inocêncio da Luz et al. (2009) reported $\mathrm{IC}_{50}$ differences among the Old and New World Leishmania species, indicating different susceptibilities to miltefosine $[L$. $(V$.) braziliensis, $L$. $(V$.) guyanensis, $L$. (L.) mexicana and Leishmania (Viannia) lainsoni]. The discrepancies between our data and these previous results may be attributed to different parasite isolates, test conditions or culture media, which are all known to affect parasitic infectivity and survival (Dey et al. 2002).

The $\mathrm{IC}_{50}$ and $\mathrm{IC}_{90}$ for $L$. (L.) donovani were $0.09 \mu \mathrm{g} / \mathrm{mL}$ $(0.22 \mu \mathrm{M})$ and $0.21 \mu \mathrm{g} / \mathrm{mL}(0.52 \mu \mathrm{M})$, respectively. These values are consistent with the published data that demonstrated an effective dose for the in vitro susceptibility of $L$. (L.) donovani clinical isolates to miltefosine ranging from $0.04 \mu \mathrm{g} / \mathrm{mL}-8.7 \mu \mathrm{g} / \mathrm{mL}(1.2 \mathrm{mM}-4.6 \mathrm{mM})$ (Escobar et al. 2002) or an $\mathrm{IC}_{50}$ of 1.5-7.1 $\mu \mathrm{M}$ (Inocêncio da Luz et al. 2009). The $\mathrm{IC}_{50}$ of miltefosine determined for $L$. (L.) donovani in this study was roughly 20 times lower than the $\mathrm{IC}_{50}$ determined for $L$. $(L$.$) chagasi (\mathrm{p}=0.03)$.

The $\mathrm{CC}_{50}$ of miltefosine against macrophages was $37.8 \mu \mathrm{g} / \mathrm{mL}(92.7 \mu \mathrm{M})$. A ratio of cytotoxicity to biological activity $\left(\mathrm{CC}_{50} / \mathrm{IC}_{50}\right)$ was used to determine the SI of miltefosine (Table). According to Weninger et al. (2001), the biological efficacy of the tested drug is not attributable to cytotoxicity when $\mathrm{SI} \geq 10$.

Parasite and host factors may determine the clinical outcome of a new drug treatment. Among the parasite factors, the inter-species intrinsic variation in drug sensitivity can be estimated by simple in vitro studies to design dose-defining clinical assays. Miltefosine was first tested against visceral leishmaniasis caused by $L$. (L.) donovani in India. According to the results of this study, one might expect that higher doses of miltefosine would be needed to treat patients infected with $L$. (L.) chagasi or the other species that are prevalent in Brazil.

\section{REFERENCES}

Bhattacharya SK, Jha TK, Sundar S, Thakur CP, Engel J, Sindermann H, Junge K, Karbwang J, Bryceson ADM, Berman JD 2004. Efficacy and tolerability of miltefosine for childhood visceral leishmaniasis in India. Clin Infect Dis 38: 217-221.

Croft SL, Snowdon D, Yardley V 1996. The activities of four anticancer alkyllsophospholipids against Leishmania donovani,
Trypanosoma cruzi and Trypanosoma brucei. J Antimicrob Chemother 38: 1041-1047.

Dey T, Afrin F, Anam K, Ali N 2002. Infectivity and virulence of Leishmania donovani promastigotes: a role for media, source and strain of parasite. J Eukaryot Microbiol 49: 270-274.

Escobar P, Matu S, Marques C, Croft SL 2002. Sensitivities of Leishmania species to hexadecylphosphocholine (miltefosine), ET-18$\mathrm{OCH}_{3}$ (edelfosine) and amphotericin B. Acta Trop 81: 151-157.

Fischer C, Voss A, Engel J 2001. Development status of miltefosine as first oral drug in visceral and cutaneous leishmaniasis. Med Microbiol Immunol 190: 85-87.

Huber W, Koella JC 1993. A comparison of three methods of estimating $\mathrm{EC}_{50}$ in studies of drugs resistance of malaria parasites. Acta Trop 55: 257-261.

Inocêncio da Luz R, Vermeersch M, Dujardin JC, Cos P, Maes L 2009. In vitro sensitivity testing of Leishmania clinical field isolates: preconditioning of promastigotes enhances infectivity for macrophages host cells. Antimicrob Agents Chemother 53: 5197-5203.

Kaminsky R 2002. Miltefosine Zentaris. Curr Opin Investig Drugs 3: $550-554$.

Le Fichoux Y, Rousseau D, Ferrua B, Ruette S, Lelièvre A, Grousson D, Kubar J 1998. Short- and long-term efficacy of hexadecylphosphocholine against established Leishmania infantum infection in BALB/c mice. Antimicrob Agents Chemother 42: 654-658.

Morais-Teixeira E de, Carvalho AS de, Costa JCS da, Duarte SL, Mendonça JS, Boechat N, Rabello A 2008. In vitro and in vivo activity of meglumine antimoniate produced at FarmanguinhosFiocruz, Brazil, against Leishmania (Leishmania) amazonensis, L. (L.) chagasi and L. (Viannia) braziliensis. Mem Inst Oswaldo Cruz 103: 358-362.

Ouellette M, Drummelsmith J, Papadopoulou B 2004. Leishmaniasis: drugs in the clinic, resistence and new developments. Drug Resist Updat 7: 257-266.

Paris C, Loiseau PM, Boris C, Bréard J 2004. Miltefosine induces apoptosis-like death in Leishmania donovani promastigotes. Antimicrob Agents Chemother 48: 852-859.

Schraner C, Hasse B, Hasse U, Baumann D, Faeh A, Burg G, Grimm F, Mathis A, Weber R, Günthard HF 2005. Successful treatment with miltefosine of disseminated cutaneous leishmaniasis in a severely immunocompromised patient infected with HIV-1. Clin Infect Dis 40: e120-e124. 
Soto J, Arana A, Toledo J, Rizzo N, Vega JC, Diaz A, Luz M, Gutierrez P, Arboleda M, Berman JD, Junge K, Engel J, Sindermann H 2004. Miltefosine for New World cutaneous leishmaniasis. Clin Infect Dis 38: 1266-1272.

Sundar S, Rosenkaimer F, Makharia MK, Goyal AK, Mandal AK, Voss A, Hilgard P, Murray HW 1998. Trial of oral miltefosine for visceral leishmaniasis. Lancet 352: 1821-1823.
Weninger B, Robledo S, Arango GJ, Deharo E, Arango R, Munoz V, Callapa J, Lobstein A, Anton R 2001. Antiprotozoal activities of Colombian plants. J Ethnopharmacol 78: 193-200.

Yardley V, Croft SL, De Doncker S, Dujardin JC, Koirala S, Rijal S, Miranda C, Llanos-Cuentas A, Chappuis F 2005. The sensitivity of clinical isolates of Leishmania from Peru and Nepal to miltefosine. Am J Trop Med Hyg 73: 272-275. 